\title{
Stochastic Viscosity Solutions for SPDEs with Discontinuous Coefficients
}

\author{
Yidong Zhang \\ College of Science, North China University of Technology, Beijing, China \\ Email: Yidong_Zhang_NCUT@163.com
}

\begin{abstract}
How to cite this paper: Zhang, Y.D. (2020) Stochastic Viscosity Solutions for SPDEs with Discontinuous Coefficients. Applied Mathematics, 11, 1219-1228.

https://doi.org/10.4236/am.2020.1111083

Received: October 15, 2020

Accepted: November 27, 2020

Published: November 30, 2020

Copyright (c) 2020 by author(s) and Scientific Research Publishing Inc.

This work is licensed under the Creative Commons Attribution International License $(\mathrm{CC}$ BY 4.0).
\end{abstract}

http://creativecommons.org/licenses/by/4.0/

\section{Open Access}

\begin{abstract}
In this paper, a class of nonlinear stochastic partial differential equations with discontinuous coefficients is investigated. This study is motivated by some research on stochastic viscosity solutions under non-Lipschitz conditions recently. By studying the solutions of backward doubly stochastic differential equations with discontinuous coefficients and constructing a new approximation function $f_{n}$ to the coefficient $f$, we get the existence of stochastic viscosity subsolutions (or super-solutions). The results of this paper can be seen as the extension and application of related articles.
\end{abstract}

\section{Keywords}

Stochastic Partial Differential Equation, Stochastic Viscosity Solution, Backward Doubly Stochastic Differential Equation

\section{Introduction}

The notion of (deterministic) viscosity solution was first introduced by Crandall and Lions [1] as an extension of the classical solutions of partial differential equations (PDEs in short). It is widely used in optimization control, differential game, font-end evolution problems and so on. The existence and uniqueness of viscosity solutions have been extensively studied in the past few decades (see e.g. [2] and [3]). Based on the fact that almost all the deterministic problems in the above applied fields have corresponding stochastic problems, Lions and Souganidis [4] introduced the notion of stochastic viscosity solutions for the first time. They used "stochastic characteristics" to eliminate the stochastic integrals in the stochastic partial differential equations (SPDEs in short), so as to solve the difficulty of defining the stochastic viscosity solutions caused by the existence of the martingale term. With the same purpose, Buckdahn and Ma [5] transformed SPDEs into ordinary PDEs with random coefficients by using a nonlinear version of Doss-Sussmann transformation, so that stochastic viscosity solutions of SPDEs can be connected with solutions of backward doubly stochastic differential equations (BDSDEs in short). 
Backward stochastic differential equations (BSDEs in short) were introduced by Pardoux and Peng [6], which have wide connections with mathematical finance and PDEs, and then they introduced a new class of BDSDEs under Lipschitz conditions to produce a probabilistic representation of certain quasilinear SPDEs in [7]. Many literatures have made efforts to obtain the existence and uniqueness results of solutions to BDSDE under relaxed Lipschitz conditions. Shi, Gu and Liu [8] proved the existence of minimum solution of BDSDE and obtained the corresponding comparison theorem under the condition that $f$ is continuous with respect to $(y, z)$ and satisfies the linear growth condition. Lin [9] got the same results under the condition that $f$ is left-continuous with respect to $y$, non-decreasing and bounded. Recently, Zhang, Xiao and Fan [10] considered the condition that $f$ is left-continuous and left-Lipschitz with respect to $y$, linear growth on $(y, z)$, and $\{f(t, 0,0)\}_{t \in[0, T]}$ is square integrable. Today, BDSDEs are one of the most useful tools in the study of SPDEs. Boufoussi, Van Casteren and Mrhardy [11] gave the existence of stochastic viscosity solutions for semi-linear SPDEs with a Neunann boundary condition by establishing the relationship between generalized BDSDEs and SPDEs as [5] did, when the coefficients are Lipschitz continuous. After that Djehiche, N'zi and Owo [12] relaxed the Lipschitz continuity conditions and considered the existence result to nonlinear SPDEs with continuous coefficients, but with Dirichlet boundary conditions.

In this paper, we consider the existence of stochastic viscosity solutions for nonlinear SPDEs with discontinuous coefficients. The nonlinear SPDEs we deal with in this paper are of the form:

$$
\begin{aligned}
u(t, x) & =l(x)+\int_{t}^{T}\left\{L u(s, x)+f\left(s, x, u(s, x), \sigma^{*}(x) D u(s, x)\right)\right\} d s \\
& +\int_{t}^{T} g(s, x, u(s, x)) \overleftarrow{d B}_{s}, t \in[0, T]
\end{aligned}
$$

where $D u$ denotes the gradient of $u ; \overleftarrow{d B}_{t}$ integral is a backward stochastic Kunita-Itô integral; $l, f, g$ are some measurable functions; $L$ is a second-order differential operator defined by

$$
L=\frac{1}{2} \sum_{i, j=1}^{d}\left(\sigma \sigma^{*}\right)_{i, j} \frac{\partial^{2}}{\partial x_{i} \partial x_{j}}+\sum_{i=1}^{d} \beta_{i} \frac{\partial}{\partial x_{i}},
$$

From [5], we can connect the SPDE (1) with the following BDSDE (2) by using a Doss-Sussmann transformation.

$$
\begin{aligned}
Y_{s}^{t} & =l\left(X_{T}^{t}\right)+\int_{s}^{T} f\left(r, X_{r}^{t}, Y_{r}^{t}, Z_{r}^{t}\right) d r \\
& +\int_{s}^{T} g\left(r, X_{r}^{t}, Y_{r}^{t}\right) \overleftarrow{d B_{r}}-\int_{s}^{T} Z_{r}^{t} d W_{r}, \\
& t \leq s \leq T,
\end{aligned}
$$

where $d W_{t}$ integral is a forward Itô integral and $\left\{X_{s}^{t} ; t \leq s \leq T\right\}$ is the solution of SDE:

$$
X_{s}^{t}(x)=x+\int_{s}^{t} b\left(X_{r}^{t}(x)\right) d r+\int_{s}^{t} \sigma\left(X_{r}^{t}(x)\right) d W_{r}, t \leq s \leq T .
$$

We prove that the minimal solution of BDSDE (2) is exactly a stochastic viscosity sub-solution of $\operatorname{SPDE}(1)$ when the coefficient $f$ 
is left continuous, non-decreasing and linear growth with respect to $(x, y, z)$. We propose a new approximation function $f_{n}$ of the coefficient $f$ such that $f_{n}$ is Lipschitz continuous with respect to y, thus solving the problems caused by discontinuity. Our work generalizes the result of [11] and of [12], which can also be seen as some applications of the results in [10].

The present paper is organized as follows. In Section 2, some preliminaries, notations and the definition of stochastic viscosity solutions are shown. The existence results of stochastic viscosity sub-solution for SPDE (1) is given in Section 3, and the conclusion is given in Section 4.

\section{Preliminaries and Definitions}

\subsection{Notations and Assumptions}

Let $\left(\Omega^{1}, F^{1}, \mathbb{P}^{1}\right)$ and $\left(\Omega^{2}, F^{2}, \mathbb{P}^{2}\right)$ are two complete probability spaces where two mutually independent Brownian motions $\left\{W_{t}: 0 \leq t \leq T\right\}$ and $\left\{B_{t}: 0 \leq t \leq T\right\}$ are defined, respectively. $T>0$ is a fixed final time. Let $F_{s, t}^{\eta}=\sigma\left\{\eta_{r}-\eta_{s}: s \leq r \leq t\right\} \vee N$ for any process $\left\{\eta_{t}\right\}$, where $N$ denotes the class of $\mathbb{P}^{i}$-null sets of $F^{i}$. Define $F_{t} \triangleq F_{t}^{W} \vee F_{t, T}^{B}$. Note that $F_{t}$ is not a filtration. Set $F^{B} \triangleq\left\{F_{t, T}^{B}\right\}_{0 \leq t \leq T}, F^{W} \triangleq\left\{F_{t, T}^{W}\right\}_{0 \leq t \leq T}$.

Let $(\Omega, F, \mathbb{P})$ denote the product space, where $\Omega=\Omega^{1} \times \Omega^{2}$, $F=F^{1} \otimes F^{2}, \mathbb{P}=\mathbb{P}^{1} \otimes \mathbb{P}^{2}$. Any random variable $\xi\left(\omega_{1}\right), \omega_{1} \in \Omega^{1}$ (resp. $\eta\left(\omega_{2}\right), \omega_{2} \in \Omega^{2}$ ) is considered as a random variable on $\Omega$ if $\xi(\omega)=\xi\left(\omega_{1}\right)$ (resp. $\left.\eta(\omega)=\eta\left(\omega_{2}\right)\right), \omega \triangleq\left(\omega_{1}, \omega_{2}\right)$. Throughout this article, we denote $\mathbb{E}$ and $\mathbb{E}_{1}$ be generic Euclidean spaces and $|\cdot|$ be the norm in $\mathbb{E} . \mathcal{M}_{0, T}^{B}$ denotes the set of $F^{B}$-stopping times $\tau$ such that $0 \leq \tau \leq T, \mathbb{P}^{2}$-a.e.

We denote $C^{k, n}\left([0, T] \times \mathbb{E} ; \mathbb{E}_{1}\right)$ to be the space of all $\mathbb{E}_{1}$-valued functions defined on $[0, T] \times \mathbb{E}$ which are $k$-times continuously $\mathrm{d}$ ifferentiable in $t$ and $n$-times continuously differentiable in $x \in \mathbb{E}$; $C_{b}^{k, n}\left([0, T] \times \mathbb{E} ; \mathbb{E}_{1}\right)$ is the subset of $C^{k, n}\left([0, T] \times \mathbb{E} ; \mathbb{E}_{1}\right)$ where all $\mathbb{E}_{1-}$ valued functions have uniformly bounded partial derivatives.

$C^{k, n}\left(F^{B},[0, T] \times \mathbb{E} ; \mathbb{E}_{1}\right)\left(\operatorname{resp} . C_{b}^{k, n}\left(F^{B},[0, T] \times \mathbb{E} ; \mathbb{E}_{1}\right)\right)$ denotes the space of random fields $\varphi \in C^{k, n}\left(F^{B},[0, T] \times \mathbb{E} ; \mathbb{E}_{1}\right)$

$\left(\right.$ resp. $\left.C_{b}^{k, n}\left(F^{B},[0, T] \times \mathbb{E} ; \mathbb{E}_{1}\right)\right)$ such that $\left(t, \omega_{2}\right) \mapsto \varphi\left(t, x, \omega_{2}\right)$ is $F^{B}$ progressively measurable for fixed $x \in \mathbb{E}$.

$\mathcal{L S C}\left([0, T] \times \mathbb{E} ; \mathbb{E}_{1}\right)\left(\right.$ resp. $\left.\mathcal{U} \mathcal{S C}\left([0, T] \times \mathbb{E} ; \mathbb{E}_{1}\right)\right)$ is the space of all $\mathbb{E}$-valued functions defined on $[0, T] \times \mathbb{E}$ which are lower (resp.upper) semi-continuous. Similarly, we can define $\mathcal{L S C}\left(F^{B},[0, T] \times \mathbb{E} ; \mathbb{E}_{1}\right)$ and $\mathcal{U S C}\left(F^{B},[0, T] \times \mathbb{E} ; \mathbb{E}_{1}\right)$

For sub- $\sigma$-field $G \in F_{T}^{B}$ and constant $p \geq 0$, let $L^{P}(G ; \mathbb{E})$ denote $G$-measurable random variables $\xi$ which are valued in $\mathbb{E}$ and satisfy $E|\xi|^{p}<\infty$.

Henceforth, for ease of notations, we denote $D=D_{x}=$ $\left(\partial / \partial x_{1}, \ldots, \partial / \partial x_{q}\right), \quad D_{y}=\partial / \partial y, \quad D_{t}=\partial / \partial t$ and $D_{x x}=$ $\left(\partial^{2} x_{i} x_{j}\right)_{i, j=1, \ldots, q}$. We have to point out that $\mathrm{K}$ is some constant, allowing differences here and there.

In this paper, we assume that $g$ always satisfies the following assumptions:

(H1) $g \in C_{b}^{0,2,3}\left([0, T] \times \mathbb{R}^{q} \times \mathbb{R} ; \mathbb{R}^{l}\right)$;

The function $f: \Omega^{2} \times[0, T] \times \mathbb{R}^{q} \times \mathbb{R} \times \mathbb{R}^{d} \mapsto \mathbb{R}$ is a random field which is discontinuous in $y$ and continuous in $z$, such that for all $\left(\omega_{2}, t, x, y, z\right) \in \Omega^{2} \times[0, T] \times \mathbb{R}^{q} \times \mathbb{R} \times \mathbb{R}^{d}, f\left(\omega_{2}, t, x, y, z\right)$ is $F_{t^{-}}$ 
measurable. Moreover, $f\left(\omega_{2}, \cdot, \cdot, 0,0\right)$ is square-integrable. We give the following assumptions:

(H2) The function $f\left(\omega_{2}, t, x, \cdot, z\right)$ is left continuous and nondecreasing;

(H3) There exists a positive-valued and $F_{t}$-measurable process $h(\cdot)$ satisfying $E\left(\int_{0}^{T}|h(t)|^{2} d t\right)<\infty$ and a positive-valued constant $K$, such that

$$
\left|f\left(\omega_{2}, t, x, y, z\right)\right| \leq h(t)+K(|x|+|y|+|z|)
$$

for each $\left(\omega_{2}, t, x, y, z\right) \in \Omega^{2} \times[0, T] \times \mathbb{R}^{q} \times \mathbb{R} \times \mathbb{R}^{d}$;

(H4) There exists a constant $K>0$ such that for all $\left(\omega_{2}, y\right) \in \Omega^{2} \times \mathbb{R}$ and $\left(t_{i}, x_{i}, z_{i}\right) \in[0, T] \times \mathbb{R}^{q} \times \mathbb{R}^{d}, i=1,2$,

$$
\begin{aligned}
& \left|f\left(\omega_{2}, t_{1}, x_{1}, y, z_{1}\right)-f\left(\omega_{2}, t_{2}, x_{2}, y, z_{2}\right)\right| \\
& \leq K\left(\left|t_{1}-t_{2}\right|+\left|x_{1}-x_{2}\right|+\left|z_{1}-z_{2}\right|\right) .
\end{aligned}
$$

(H5) The functions $\sigma: \mathbb{R}^{q} \mapsto \mathbb{R}^{q \times q}$ and $\beta: \mathbb{R}^{q} \mapsto \mathbb{R}$ are uniformly Lipschitz continuous with Lipschitz constant $K>0$.

(H6) $l(x)$ is a continuous function such that for some $p>0$, it satisfies

$$
|l(x)| \leq K\left(1+|x|^{p}\right), x \in \mathbb{R}^{q} .
$$

\subsection{Definition of Stochastic Viscosity Solutions}

In the light of the definition of stochastic viscosity solutions in [5], the following stochastic flow $\eta \in C^{0,0,0}\left(F^{B},[0, T] \times \mathbb{R}^{q} \times \mathbb{R} ; \mathbb{R}\right)$, which is the unique solution of the following BSDE in the Stratonovich sense, plays an important role.

$$
\eta(t, x, y)=y+\int_{t}^{T} g(s, x, \eta(s, x, y)) \circ \overleftarrow{d B_{s}}
$$

We can rewrite BSDE (3) to its equivalent Itô form:

$$
\begin{aligned}
\eta(t, x, y) & =y+\frac{1}{2} \int_{t}^{T} g(s, x, \eta(s, x, y)) D_{y} g(s, x, \eta(s, x, y)) d s \\
& +\int_{t}^{T} g(s, x, \eta(s, x, y)) \overleftarrow{d B}_{s}, 0 \leq t \leq T,
\end{aligned}
$$

where the mapping $y \mapsto \eta(t, x, y)$ is a diffeomorphism under assumption (H1). Therefore, let $E(t, x, y)$ denote the $y$-inverse of $\eta(t, x, y)$. We can get easily that $E(t, x, y)$ is the solution of the following BSDE:

$$
E(t, x, y)=y-\int_{t}^{T} D_{y} E(s, x, y) g(s, x, y) \circ \overleftarrow{d B}_{s}
$$

For any random field $\psi:[0, T] \times \mathbb{R}^{q} \mapsto \mathbb{R}$, we can introduce the transformation

$$
\varphi(t, x)=E(t, x, \psi(t, x)),
$$

namely $\psi(t, x)=\eta(t, x, \varphi(t, x))$, then $\psi \in C^{0, p}\left(F^{B},[0, T] \times \mathbb{R}\right)$ if and only if $\psi \in C^{0, p}\left(F^{B},[0, T] \times \mathbb{R}^{q} ; \mathbb{R}\right)$, for $p=0,1,2$.

Applying Itô's formula to $\psi(t, x)$, it follows that

$$
\begin{aligned}
& \psi(t, x)=\eta(t, x, \varphi(t, x)) \\
& =\varphi(T, x)+\frac{1}{2} \int_{t}^{T} g\left(s, x, \eta(s, x, \varphi(s, x)) D_{y} g(s, x, \eta(s, x, \varphi(s, x)) d s\right. \\
& +\int_{t}^{T} g(s, x, \eta(s, x, y)) \overleftarrow{d B}_{s}-\int_{t}^{T} D_{y} \eta(s, x, \varphi(s, x)) D_{t} \varphi(s, x) d s
\end{aligned}
$$


According to the discussion on constructing stochastic viscosity solution in [5], it is natural to compare

$$
L \psi(t, x)+f\left(t, x, \psi(t, x), \sigma^{*}(x) D \psi(t, x)\right)
$$

and

$\frac{1}{2} g\left(t, x, \eta(t, x, \varphi(t, x)) D_{y} g\left(t, x, \eta(t, x, \varphi(t, x))-D_{y} \eta(t, x, \varphi(t, x)) D_{t} \varphi(t, x)\right.\right.$.

Thus, we give the following definition:

Definition. A random field

$u \in \mathcal{L S C}\left(F^{B},[0, T] \times \mathbb{R}^{q} ; \mathbb{R}\right)\left(\operatorname{resp} . \mathcal{U S C}\left(F^{B},[0, T] \times \mathbb{R}^{q} ; \mathbb{R}\right)\right)$ is called a stochastic viscosity sub-solution (resp. super-solution) of SPDE (1) if $u(T, x) \leq l(x)$ (resp. $u(T, x) \geq l(x)$ ), for all $x \in \mathbb{R}^{q}$ and if for any $\tau \in \mathcal{M}_{0, T}^{B}, \xi \in L^{0}\left(F_{\tau}^{B} ; \mathbb{R}^{q}\right)$ and $\varphi \in C^{1,2}\left(F_{\tau}^{B},[0, T] \times \mathbb{R}^{q} ; \mathbb{R}\right)$ satisfying

$$
\begin{aligned}
& u\left(\omega_{2}, t, x\right)-\eta\left(\omega_{2}, t, x, \varphi(t, x)\right) \leq(\text { resp. } \geq) 0 \\
& =u\left(\tau\left(\omega_{2}\right), \xi\left(\omega_{2}\right)\right)-\eta\left(\tau\left(\omega_{2}\right), \xi\left(\omega_{2}\right), \varphi\left(\tau\left(\omega_{2}\right), \xi\left(\omega_{2}\right)\right)\right.
\end{aligned}
$$

for all $(t, x)$ in a neighborhood of $(\tau, \xi), \mathbb{P}^{2}$-a.e. Then, on $\{0<\tau<T\}$,

$$
\begin{aligned}
& L \psi(\tau, \xi)+f\left(\tau, \xi, \psi(\tau, \xi), \sigma^{*}(\xi) D \psi(\tau, \xi)\right) \geq(\text { resp. } \leq) \\
& \frac{1}{2} g(\tau, \xi, \psi(\tau, \xi)) D_{y} g(\tau, \xi, \psi(\tau, \xi))-D_{y} \eta(\tau, \xi, \varphi(\tau, \xi)) D_{t} \varphi(\tau, \xi),
\end{aligned}
$$

where $\psi(t, x) \triangleq \eta(t, x, \varphi(t, x))$.

\section{Existence of Stochastic Viscosity Sub-Solutions (or Super-Solutions)}

We are now in position to discuss the existence of stochastic viscosity sub-solutions of SPDE (1). To begin with ,it is indispensable to introduce two sets that are widely used in BDSDE study, such as [7].

For any $n \in \mathbb{N}, M^{2}\left(0, T ; \mathbb{R}^{n}\right)$ denotes the set of $(d P \times d t$ a.e. equal) $n$ dimensional jointly measurable random processed $\left\{\varphi_{t}: 0 \leq t \leq T\right\}$ satisfying

- $E \int_{t}^{T}\left|\varphi_{t}\right|^{2} d t<\infty$;

- $\varphi_{t}$ is $F_{t}$-measurable for a.e. $t \in[0, T]$.

Let $S^{2}\left([0, T] ; \mathbb{R}^{n}\right)$ denote the set of continuous $n$ dimensional random processes satisfying:

- $E\left(\sup _{0 \leq t \leq T}\left|\varphi_{t}\right|^{2}\right)<\infty$;

- $\varphi_{t}$ is measurable for any $t \in[0, T]$.

We consider the following two BDSDEs:

$$
\begin{aligned}
Y_{s}^{t, 1} & =l\left(X_{T}^{t}\right)+\int_{s}^{T} f\left(r, X_{r}^{t}, Y_{r}^{t, 1}, Z_{r}^{t, 1}\right) d r+\int_{s}^{T} g\left(r, X_{r}^{t}, Y_{r}^{t, 1}\right) \overleftarrow{d B_{r}} \\
& -\int_{s}^{T} Z_{r}^{t, 1} d W_{r}, t \leq s \leq T
\end{aligned}
$$




$$
\begin{aligned}
Y_{s}^{t, 2} & =l\left(X_{T}^{t}\right)+\int_{s}^{T}\left[h(r)+K\left(\left|X_{r}^{t}\right|+\left|Y_{r}^{t, 2}\right|+\left|Z_{r}^{t, 2}\right|\right)\right] d r \\
& +\int_{s}^{T} g\left(r, X_{r}^{t}, Y_{r}^{t, 2}\right) \overleftarrow{d B_{r}}-\int_{s}^{T} Z_{r}^{t, 2} d W_{r}, t \leq s \leq T
\end{aligned}
$$

where the function $f$ in (4) satisfies assumptions (H2)-(H4), and $g$ in both (4) and (5) satisfy assumption (H1).

By Theorem 3.1 in [10], BDSDE (4) has at least one solution. Moreover, there exists a minimal solution $\left(\underline{Y}_{s}^{t, 1}, \underline{Z}_{s}^{t, 1}\right)$ of BDSDE (4) in the sense that for any other solution $\left(Y_{s}^{t, 1}, Z_{s}^{t, 1}\right)$ of $(4)$, we have $\underline{Y}_{s}^{t, 1} \leq Y_{s}^{t, 1}, t \leq s \leq T$.

It is easy to check that BDSDE (4) and BDSDE (5) meet the conditions of the comparison theorem in [10] under assumptions (H1)-(H5), then if we denote the minimal solutions of BDSDE (4) and (5) by $\left(\underline{Y}_{s}^{t, 1}, \underline{Z}_{s}^{t, 1}\right)$ and $\left(\underline{Y}_{s}^{t, 2}, \underline{Z}_{s}^{t, 2}\right)$, respectively, we have $\underline{Y}_{s}^{t, 1} \leq \underline{Y}_{s}^{t, 2}$. Thus we can turn to study the boundedness of $\underline{Y}_{s}^{t, 2}$ rather than $\underline{Y}_{s}^{t, 1}$ directly. On the boundedness of $\underline{Y}_{s}^{t, 2}$, we have the following lemma:

Lemma 1. There exists a constant $B>0$ depending on $K, T$ and $l\left(X_{T}^{t}\right)$ such that $E\left(\sup _{0 \leq s \leq T}\left|\underline{Y}_{s}^{t, 2}\right|^{2}\right) \leq B$.

Proof. The proof of this lemma is parallel to that of Lemma 4.2 in [8]. So we omit it.

In addition, the following lemma is necessary to prove stochastic viscosity sub-solution.

Lemma 2. $\left\{f_{n}\right\}_{n \geq 0}$ is a sequence of functions defined by

$$
f_{n}=\inf _{u \in \mathbb{R}}\left\{n \int_{u-\frac{1}{n}}^{u} f(t, x, r, z) d r+n K|y-u|\right\} .
$$

Then it follows that

(i) $\left|f_{n}(t, x, y, z)\right| \leq h(t)+K(|x|+|y|+|z|)$;

(ii) $\left|f_{n}\left(t, x, y_{1}, z\right)-f_{n}\left(t, x, y_{2}, z\right)\right| \leq n K\left|y_{1}-y_{2}\right|$;

(iii) $\mid f_{n}\left(t_{1}, x_{1}, y, z_{1}\right)-f_{n}\left(t_{2}, x_{2}, y, z_{2}\right) \leq K\left(\left|t_{1}-t_{2}\right|+\left|x_{1}-x_{2}\right|+\mid z_{1}-\right.$ $\left.z_{2} \mid\right)$

(iv) if sequence $y_{n}$ increases to $y$, then $\lim _{n \rightarrow \infty} f_{n}\left(\omega_{2}, t, x, y_{n}, z\right)=$ $f\left(\omega_{2}, t, x, y, z\right)$, a.s..

Proof. The proof of this lemma is mainly inspired by [13] and [14]. Indeed, $f_{n} \leq f \leq h(t)+K(|x|+|y|+|z|)$ is obvious, and on the other hand, we obtain

$$
\begin{aligned}
f_{n} & \left.\geq \inf _{u}\left\{n \int_{u-\frac{1}{n}}^{u}(-h(t)-K(|x|+|r|+|z|)) d r+n K|y-u|\right)\right\} \\
& \geq \inf _{u}\left\{-h(t)-K(|x|+|z|)-n K \int_{u-\frac{1}{n}}^{u}|r| d r+n K|y-u|\right\} \\
& \geq \inf _{u}\{-h(t)-K(|x|+|u|+|z|)+K|y-u|\} \\
& \geq-h(t)-K(|x|+|y|+|z|) .
\end{aligned}
$$

Thus, (i) holds. From the definition of $f_{n}$ and inequality $\left|\inf _{x \in D} f_{1}(x)-\inf _{x \in D} f_{2}(x)\right| \leq \sup _{x \in D}\left|f_{1}(x)-f_{2}(x)\right|$, we can readily 
get (ii). (iii) is an immediate result of (H4). Now we start to prove (iv). In the light of the definition of $f_{n}$, we can deduce that there exists a sequence $\left\{u_{n}\right\}$ which converges to $y$ such that $d P \times d t$-a.e.

$$
\begin{aligned}
& n \int_{u_{n}-\frac{1}{n}}^{u_{n}} f(t, x, r, z) d r+n K\left(\left|y_{n}-u_{n}\right|\right)-\frac{1}{n} \\
& \leq f_{n}\left(t, x, y_{n}, z\right) \leq f\left(t, x, y_{n}, z\right) .
\end{aligned}
$$

By Lemma 3.1 in [14], we obtain

$$
\lim _{y_{n} \rightarrow y} n \int_{y_{n}-\frac{1}{n}}^{y_{n}} f(t, x, r, z) d r=f(t, x, y, z)
$$

for any fixed $y \in \mathbb{R}$. Moreover, because of the left continuity of $f$, we obtain that $\lim _{y_{n} \rightarrow y} f\left(t, x, y_{n}, z\right)=f(t, x, y, z)$. Taking the limit on both sides of (6), we get $\lim _{n \rightarrow \infty} f_{n}\left(t, x, y_{n}, z\right)=f(t, x, y, z)$, for which (iv) holds.

Now, we are going to verify that $\underline{Y}_{t}^{t, 1}$ is a stochastic viscosity subsolution of SPDE (1).

Lemma 3. Let $u_{*}: \Omega^{2} \times[0, T] \times \mathbb{R}^{q} \mapsto \mathbb{R}$ be a random field defined by

$$
u_{*}(t, x) \triangleq \underline{Y}_{t}^{t, 1}
$$

for all $(t, x) \in[0, T] \times \mathbb{R}^{q}$. Then $u_{*} \in \mathcal{L S C}\left(F^{B},[0, T] \times \mathbb{R}^{q} ; \mathbb{R}\right)$.

Proof. Let $\left(f_{n}\right)_{n \leq 1}$ be the non-decreasing sequence of Lipschitz continuous functions as defined in Lemma 2 to approximate $f$.

Let $\left(Y_{s}^{n, t}, Z_{s}^{n, t}, t \leq s \leq T\right)$ be the solution of the following BDSDE:

$$
\begin{aligned}
Y_{s}^{n, t} & =l\left(X_{T}^{t}\right)+\int_{s}^{T} f_{n}\left(r, X_{r}^{t}, Y_{r}^{n, t}, Z_{r}^{n, t}\right) d r+\int_{s}^{T} g\left(r, X_{r}^{t}, Y_{r}^{n, t}\right) \overleftarrow{d B_{r}} \\
& -\int_{s}^{T} Z_{r}^{n, t} d W_{r} .
\end{aligned}
$$

Similar to the proof of Theorem 3.1 in [10], we can verify that $\left(Y_{s}^{n, t}, Z_{s}^{n, t}\right)$ converges to $\left(\underline{Y}_{s}^{t, 1}, \underline{Z}_{s}^{t, 1}\right)$ which is the minimal solution of BDSDE (4).

Let $u_{n}:[0, T] \times \mathbb{R}^{q} \mapsto \mathbb{R}$ be a random fields defined by

$$
u_{n}(t, x)=Y_{t}^{n, t},(t, x) \in[0, T] \times \mathbb{R}^{q} .
$$

It is obvious that $\left(u_{n}\right)_{n \geq 1}$ is a non-decreasing sequence and it converges to $u_{*}$. Thus, $u_{*} \in \mathcal{L} \mathcal{L C}\left(F^{B},[0, T] \times \mathbb{R}^{q} ; \mathbb{R}\right)$.

Remark. BDSDE (4) has a maximal solution $\bar{Y}_{s}^{t, 1}$ when the coefficient $f$ is right continuous and non-decreasing. Using the similar proof of Lemma 3 where $f_{n}$ is replaced by $f_{n} \triangleq \sup _{u \in \mathbb{R}}\left\{n \int_{u}^{u+\frac{1}{n}} f(t, x, r, z)-\right.$ $n K|y-u|\}$, we can prove that $u^{*}(t, x) \triangleq \bar{Y}_{t}^{t, 1}$ satisfies $u^{*} \in$ $\mathcal{U S C}\left(F^{B},[0, T] \times \mathbb{R}^{q} ; \mathbb{R}\right)$.

Next, we will state our main result. The technique of Theorem 3.2 in [12] is used in the proof of the following theorem. But for sake of the readers' convenience, we still give a short proof. 
Theorem 4. Under assumptions (H1)- (H6), the random field $u_{*} \in$ $\mathcal{L S C}\left(F^{B},[0, T] \times \mathbb{R}^{q} ; \mathbb{R}\right)$ defined by $u_{*} \triangleq \underline{Y}_{t}^{t, 1}$ is a stochastic viscosity sub-solution for $\operatorname{SPDE}(1)$. Further, for each classical solution $v \in$ $C^{0,2}\left(F^{B},[0, T] \times \mathbb{R}^{q} ; \mathbb{R}\right)$ of $\operatorname{SPDE}(1)$, we have $u_{*}(t, x) \leq v(t, x)$.

Proof. For all $x \in \mathbb{R}^{q}$, we readily have that $u(T, x) \leq l(x)$. Now, we consider under the condition that if for any $\tau \in M_{0, T}^{B}, \xi \in L^{0}\left(F_{T}^{B} ; \mathbb{R}^{q}\right)$ and $\varphi \in C^{1,2}\left(F_{\tau}^{B},[0, T] \times \mathbb{R}^{\prime \prime} ; \mathbb{R}\right)$ such that

$$
u_{*}(t, x)-\eta(t, x, \varphi(t, x)) \leq 0=u_{*}(\tau, \xi)-\eta(\tau, \xi, \varphi(\tau, \xi))
$$

for all $(t, x)$ in a neighborhood of $(\tau, \xi), \mathbb{P}^{2}$-a.e. It is not hard to check that BDSDE (7) meets the conditions in [11] under assumptions (H1) - (H6). Thus, $u_{n}$ is a stochastic viscosity solution for SPDE:

$$
\begin{aligned}
u(t, x) & =l(x)+\int_{t}^{T}\left\{L u(s, x)+f_{n}\left(s, x, u(s, x), \sigma^{*}(x) D u(s, x)\right\} d s\right. \\
& +\int_{t}^{T} g(s, x, u(s, x)) \overleftarrow{d B_{s}}, t \in[0, T]
\end{aligned}
$$

Therefore, if $\tau_{n} \in M_{0, T}^{B}, \xi_{n} \in L^{0}\left(F_{\tau}^{B}, \mathbb{R}^{q}\right)$ and $\varphi_{n} \in C^{1,2}\left(F_{T}^{B},[0, T] \times\right.$ $\left.\mathbb{R}^{q} ; \mathbb{R}\right)$ satisfying

$$
u_{n}(t, x)-\eta\left(t, x, \varphi_{n}(t, x)\right) \leq 0=u_{n}\left(\tau_{n}, \xi_{n}\right)-\eta\left(\tau_{n}, \xi_{n}, \varphi_{n}\left(\tau_{n}, \xi_{n}\right)\right),
$$

for each $(t, x)$ in a neighborhood of $\left(\tau_{n}, \xi_{n}\right), \mathbb{P}^{2}$-a.s.,such that $\left(\tau_{n}, \xi_{n}, \varphi_{n}\right) \rightarrow(\tau, \xi, \varphi)$. It follows that $\mathbb{P}^{2}$-a.s.

$$
\begin{aligned}
& L \psi_{n}\left(\tau_{n}, \xi_{n}\right)+f_{n}\left(\tau_{n}, \xi_{n}, \psi_{n}\left(\tau_{n}, \xi_{n}\right), \sigma^{*}\left(\xi_{n}\right) D \psi_{n}\left(\tau_{n}, \xi_{n}\right)\right) \\
& \geq \frac{1}{2} g\left(\tau_{n}, \xi_{n}, \psi_{n}\left(\tau_{n}, \xi_{n}\right)\right) D_{y} g\left(\tau_{n}, \xi_{n}, \psi_{n}\left(\tau_{n}, \xi_{n}\right)\right) \\
& -D_{y} \eta\left(\tau_{n}, \xi_{n}, \varphi_{n}\left(\tau_{n}, \xi_{n}\right)\right) D_{t} \varphi_{n}\left(\tau_{n}, \xi_{n}\right)
\end{aligned}
$$

where $\psi_{n}(t, x)=\eta\left(t, x, \varphi_{n}(t, x)\right)$ converges to $\psi(t, x)=\eta(t, x, \varphi(t, x))$ in $[0, T] \times \mathbb{R}^{q}$. Sending $n \rightarrow \infty$ in (8), we get

$$
\begin{aligned}
& L \psi(\tau, \xi)+f\left(\tau, \xi, \psi(\tau, \xi), \sigma^{*}(\xi) D \psi(\tau, \xi)\right) \\
& \geq \frac{1}{2} g(\tau, \xi, \psi(\tau, \xi)) D_{y} g(\tau, \xi, \psi(\tau, \xi))-D_{y} \eta(\tau, \xi, \varphi(\tau, \xi)) D_{t} \varphi(\tau, \xi) .
\end{aligned}
$$

Consequently, $u_{*} \in \mathcal{L} \mathcal{S C}\left(F^{B},[0, T] \times \mathbb{R}^{q} ; \mathbb{R}\right)$ is a stochastic viscosity sub-solution of SPDE (1). From [7], $\left\{v\left(s, X_{s}^{t}\right),\left(\sigma^{*} D v\right)\left(s, X_{s}^{t}\right), 0 \leq s \leq\right.$ $t \leq T\}$ is a solution of $\operatorname{BDSDE}(4)$, so $v\left(s, X_{s}^{t}\right) \geq \underline{Y}_{s}^{t, 1}$. Specially, we have $v(t, x) \geq u_{*}(t, x)$.

Remark. Analogously, if the coefficient $f$ is right continuous and non-decreasing, then $u^{*}(t, x) \triangleq \bar{Y}_{t}^{t, 1}$ is a stochastic viscosity supersolution for $\operatorname{SPDE}(1)$. Moreover, for each classical solution $v^{\prime} \in$ $C^{0,2}\left(F^{B},[0, T] \times \mathbb{R}^{\prime \prime} ; \mathbb{R}\right)$ of $\operatorname{SPDE}(1)$, we have $u^{*}(t, x) \geq v^{\prime}(t, x)$.

Finally, we give a corollary about the boundedness of $u_{*}(t, x)$ which is an immediate result of Lemma 1.

Corollary 1. Let $u_{*}(t, x) \in \mathcal{L} \mathcal{S C}\left(F^{B},[0, T] \times \mathbb{R}^{q} ; \mathbb{R}\right)$ be a stochastic viscosity sub-solution of $\operatorname{SPDE}(1)$, then there exists a constant $A>0$ such that $E\left(u_{*}(t, x)\right) \leq A$. 


\section{Conclusion}

This paper is devoted to the study of stochastic viscosity solutions to SPDE (1) whose coefficient $f$ is left (resp. right) continuous, nondecreasing with respect to $y$ and linear growth with respect to $(x, y, z)$. We present a new function $f_{n}=\inf _{u \in \mathbb{R}}\left\{n \int_{u-\frac{1}{n}}^{u} f(t, x, r, z) d r+\right.$ $n K|y-u|\} \quad\left(\right.$ resp. $\left.\quad f_{n} \triangleq \sup _{u \in \mathbb{R}}\left\{n \int_{u}^{u+\frac{1}{n}} f(t, x, r, z)-n K|y-u|\right\}\right)$ which satisfies Lipschitz conditions to approximate $f$, thus solving the trouble caused by the discontinuous coefficient. We get that the minimal (resp. maximal) solution of BDSDE (2) is a stochastic viscosity sub-solution (resp. super-solution) of SPDE (1) under given conditions. The results obtained in this paper extend the existence conditions of stochastic viscosity solutions to more relaxed conditions and generalize some results in related literature.

\section{Acknowledgements}

This work is supported by Yuyou Project of North University of Technology (No. 207051360020XN140/007) and Scientific Research Foundation of North University of Technology (No. 110051360002).

\section{Conflicts of Interest}

The author declares no conflicts of interest regarding the publication of this paper.

\section{References}

[1] Crandall, M.G. and Lions, P.L. (1983) Viscosity Solutions of Hamilton-Jacobi Equations. Transactions of the American Mathematical Society, 277, 1-42.

https://doi.org/10.1090/S0002-9947-1983-0690039-8

[2] Ishii, H. (1989) On Uniqueness and Existence of Viscosity Solutions of Fully Nonlinear Second-Order Elliptic PDE's. Communications on Pure and Applied Mathematics, 42, 15-45.

https://doi.org/10.1002/cpa.3160420103

[3] Crandall, M.G., Ishii, H. and Lions, P.L. (1992) User's Guide to Viscosity Solutions of Second Order Partial Differential Equations. Bulletin of the American Mathematical Society, 27, 1-67. http://doi.org/10.1090/S0273-0979-1992-00266-5

[4] Lions, P.L. and Souganidis, P.E. (1998) Fully Nonlinear Stochastic Partial Differential Equations. Comptes Rendus de l'Académie des Sciences. Series I. Mathematics, 326, 1085-1092. https://doi.org/10.1016/S0764-4442(98)80067-0

[5] Buckdahn, R. and Ma, J. (2001) Stochastic Viscosity Solutions for Nonlinear Stochastic Partial Differential Equations. Part I. Stochastic Processes and Their Applications, 93, 181-104. https://doi.org/10.1016/S0304-4149(00)00093-4

[6] Pardoux, E. and Peng, S. (1990) Adapted Solution of a Backward Stochastic Differential Equation. Systems and Control Letters, 14, 55-61. https://doi.org/10.1016/0167-6911(90)90082-6 
[7] Pardoux, E. and Peng, S. (1994) Backward Doubly Stochastic Differential Equations and Systems of Quasilinear SPDEs. Probability Theory and Related Fields, 98, 209-227.

https://doi.org/10.1007/BF01192514

[8] Shi, Y., Gu, Y. and Liu, K. (2005) Comparison Theorems of Backward Doubly Stochastic Differential Equations and Applications. Stochastic Analysis and Applications, 23, 97-110. https://doi.org/10.1081/SAP-200044444

[9] Lin, Q. (2009) A Class of Backward Doubly Stochastic Differential Equations with Non-Lipschitz Coefficients. Statistics and Probability Letters, 79, 2223-2229.

https://doi.org/10.1016/j.spl.2009.07.019

[10] Zhang, J., Xiao, L. and Fan, S. (2015) Existence and Comparison Theorem of Minimum Solutions for Backward Doubly Stochastic Differential Equations with Discontinuous Generators. Mathematica Applicata, 28, 909-916. (In Chinese) https://doi.org/10.13642/j.cnki.42-1184/o1.2015.04.025

[11] Boufoussi, B., Van Casteren, J. and Mrhardy, N. (2007) Generalized Backward Doubly Stochastic Differential Equations and SPDEs with Nonlinear Neumann Boundary Conditions. Bernoulli, 13, 423-446. https://doi.org/10.3150/07-BEJ5092

[12] Djehiche, B., N'zi, M. and Owo, J. (2011) Stochastic Viscosity Solutions for SPDEs with Continuous Coefficients. Journal of Mathematical Analysis and Applications, 384, 63-69.

https://doi.org/10.1016/j.jmaa.2010.08.005

[13] Lepeltier, J.P. and San Martin, J. (1997) Backward Stochastic Differential Equations with Continuous Coefficient. Statistics and Probability Letters, 32, 425-430. https://doi.org/10.1016/S0167-7152(96)00103-4

[14] Boufoussi, B. and Ouknine, Y. (2003) On a SDE Driven by a Fractional Brownian Motion and with Monotone Drift. Electronic Communications in Probability, 8, 112-134.

http://doi.org/10.1214/ecp.v8-1084 